\title{
Sequential Trials of Fluoxetine, Phenelzine, and Tranylcypromine in the Treatment of Obsessive-Compulsive Disorder
}

\author{
JACK G. MODELl, M.D., 'JOSEPH Himle, M.S.W., ${ }^{1}$ \\ Randolph M. Nesse, M.D., ${ }^{1}$ James M. Mountz, M.D., Ph.D., 2 \\ STEPHEN SCHMALTZ, M.P.H. ${ }^{3}$ \\ ${ }^{1}$ Department of Psychiatry; ${ }^{2}$ Department of Nuclear Medicine; ${ }^{3}$ Clinical Research Center, \\ Department of Medicine: University of Michigan Medical Center; Ann Arbor
}

\begin{abstract}
The effects of tluoxetine, phenelzine, and tranylcypromine in obsessive-compulsive disorder (OCD) were compared in an open-label pilot study involving sequential treatment with these agents in six patients. Despite full (or maximally tolerated) trials on these medications, there was no improvement in OC symptoms referable to the pharmacotherapy. Three patients, however, subsequently showed major improvement in symptoms following application of behavioral therapy techniques.

Despite the small sample size, this study suggests that there may be many patients with OCD for whom these medications might be poorly tolerated or ineffective, and emphasizes that behavioral-therapeutic techniques can be effective in patients for whom medications prove ineffective or intolerable. Of note, one patient displayed simultaneous manic and $O C$ symptoms, thus undermining previous suggestions that the coexistence of these symptoms may not be possible.
\end{abstract}

Several studies have found the serotonin reuptake inhibitor fluoxetine to be effective in the treatment of patients with obsessive-compulsive disorder (OCD) (Fontaine \& Chouinard, 1986; Sommi, Crimson, \& Bowden, 1987; Turner, Jacob, Beidel, \& Himmelhoch, 1985), and several case reports suggest that the monoamine oxidase inhibitors (MAOIs) phenelzine and tranylcypromine may also be helpful in some patients

\footnotetext{
The authors wish to thank Cynthia S. Pomerleau, M.A., Ph.D., for her valuable editorial assistance.

Address correspondence and reprints to: Jack G. Modell, M.D., Department of Psychiatry, University of Michigan Hospital, Room 8806, Box 0116, 1500 East Medical Center Drive, Ann Arbor, MI 48109-0116.
} 
with OCD (Jenike, 1981; Jenike, Surman, Cassem, Zusky, \& Anderson, 1983). Notably, the one double-blind, controlled study using an MAOI (Clorgyline) for the treatment of this disorder found it to be largely ineffective (Insel, Murphy, \& Cohen, 1983). The present study was intended to address the following questions: 1) Can a positive therapeutic response to phenelzine and tranylcypromine in OCD be shown prospectively? 2) Is the response rate to the MAOIs comparable to that of the neurochemically more selective agent fluoxetine? and, 3) Is there a difference in side effects and patient tolerance among these medications?

\section{METHOD}

The study population was recruited from outpatients at the University of Michigan Anxiety Disorders Clinic who presented for treatment of OCD; informed consent was obtained from all subjects. Six subjects (five female, one male: mean age 31.7; range $21-40$ ) were studied. Mean duration of illness was 13.0 years (range 6-20).

Study subjects were required to: a) fulfill the DSM-III-R criteria for OCD as determined in a semi-structured interview for Axis I disorders by a faculty psychiatrist (JGM); b) have a pre-treatment score of 10 or greater on either the obsessive or the compulsive subscales of the YaleBrown OC Scale (Goodman, Price, Rasmussen, Mazure, Delgado, et al., in press; Goodman, Price, Rasmussen, Mazure, Fleischmann, et al., in press) (Y-BOCS; see below); c) have a Hamilton depression rating on items $1-20$ of less than 10 (nondepressed); and, d) have no history of treatment with monoamine oxidase inhibitors, clomipramine, or fluoxetine. Exclusion criteria included: a) concurrent medical illness, pregnancy, or laboratory abnormality; b) use of any other psychoactive medication during the study or the two weeks preceding the study; and, c) any concurrent DSM-III-R Axis I disorder. No other therapies directed at the OC symptoms were permitted during the period of study.

The Y-BOCS, items 1-5 (obsessional subscale) and 6-10 (compulsive subscale), was completed every two weeks throughout the study. Each subscale total can range from 0 (no symptoms) to 20 (severe and incapacitating symptoms) (Goodman, Price, Rasmussen, Mazure, Fleischmann, et al., in press). Pretreatment $\mathrm{Y}$-BOCS item 1-10 scores averaged 21.8 (range $11-35$ ): subscale scores averaged 8.0 (range $0-18$ ) for obsessional symptoms and 13.8 (range 11-17) for compulsive symptoms. Also completed every two weeks throughout the study were the observer-rated portion of the Comprehensive Psychiatric Rating Scale (CPRS-O); a 35-item medication side effect inventory; a clinical interview for global assessment of symptoms and related impairment; orthostatic blood pressures and heart rates; and weight. A blood survey (CBC) and liver function tests were performed every six weeks throughout the study. An electrocardiogram and thyroid stimulating hor- 
mone assay were performed upon entry into the study. All screening laboratory tests were normal.

The study was conducted as a non-blinded, prospective medication trial in which the order of treatment with each of the three agents was randomly varied. Each patient began treatment at the first of four sequential dosage levels, as outlined in Table 1. Each patient was re-evaluated every two weeks for consideration of dosage increase as follows. Patients who showed minimal or no improvement in target symptoms, as defined by a $<20 \%$ decrease in the previous $\mathrm{Y}-\mathrm{BOCS}$ item $1-10$ total score, received a dosage increase to the next dosing level of the current medication, barring the development of any contraindications to continued drug use or dosage increase; otherwise the current medication dosage was maintained. Thus, at two-week intervals, stepwise increases through dosage levels II-IV were considered.

Inadequate response to a given agent was defined as a $<20 \%$ decrease in the previous $\mathrm{Y}-\mathrm{BOCS}$ item $1-10$ total score after two weeks on the level IV (or maximally tolerated) dosage. Patients thus considered to be inadequate drug-responders or drug-intolerant underwent medication taper and discontinuation. After a two week drug-free period, the patient received the next drug in the pre-determined sequence, following the same protocol as above. Pill-counts were performed at every visit to document medication compliance. Patients remained on an MAOI diet (Folks, 1983) throughout treatment with all medications.

Data were analyzed using a repeated measures analysis of variance for unbalanced data sets (sample variances were homogeneous); Fisher's Test for least significant differences was utilized where treatment effects were found to be significant.

\section{RESULTS}

Of the six patients who entered the study, four completed all three medication trials. Within the 15 patient-by-medication trials completed. no patient reported subjective improvement in symptoms following a full (or maximally tolerated) trial of any of the three medications. Consistent with these subjective impressions, analysis of the $\mathrm{Y}-\mathrm{BOCS}$ data showed

TABLE 1

Dosage SChedUles (MG)

\begin{tabular}{lcccccccc}
\hline & \multicolumn{2}{c}{ Fluoxetine } & & \multicolumn{2}{c}{ Phenelzine } & & \multicolumn{2}{c}{ Tranylcypromine } \\
\cline { 2 - 3 } Level & Q AM & Q Midday & & Q AM & Q Midday & & Q AM & Q Midday \\
\hline I & 20 & - & & 30 & - & & 10 & - \\
II & 20 & 20 & & 30 & 30 & & 10 & 10 \\
III & 40 & 20 & & 60 & 30 & & 20 & 10 \\
IV & 40 & 40 & & 60 & 60 & & 20 & 20 \\
\hline
\end{tabular}


that no drug treatment produced a statistically significant reduction in either the total or subscale $\mathrm{Y}-\mathrm{BOCS}$ scores for the group as a whole, or after exclusion of cases with drug-intolerance. We ceased recruiting additional patients for the study because of this apparent negative treatment outcome. A significant downward drift in total (item 1-10) Y-BOCS scores from the start to the completion of the study, however, was observed ( $p<.05$; mean change of $-23 \%$ over 22.2 weeks), but bore no correlation with the presence or absence of any specific medication.

Significant differences in some of the side effects and physiologic parameters among medications were observed. These included symptomatic orthostatic decreases in systolic and diastolic blood pressures (mean decrease $10 \mathrm{mmHg} ; p<.05$ ); increases in heart rate (mean increase 12 beats/min; $p<.05$ ) with both MAOIs; and significant $(p<.01)$ sedation and dizziness in all patients receiving phenelzine, a finding paralleled by a significant increase in observer-rated sleepiness during phenelzine administration $(p<.01)$. No significant changes were seen in laboratory values on any medication.

Five of the six patients completed the full trial of at least two weeks on $80 \mathrm{mg} /$ day fluoxetine (mean duration on fluoxetine: 8.5 weeks, range 6-12 weeks). One patient was withdrawn from fluoxetine after two weeks on $60 \mathrm{mg} /$ day due to painful abdominal bloating and gastric reflux.

Among the five patients who received phenelzine, the average dosage was $48 \mathrm{mg}$ /day (range 30-90); average duration of medication administration was 3.8 weeks (range 1-8). Intolerable side effects eventually necessitated phenelzine discontinuation in one patient following one week of $30 \mathrm{mg} /$ day, in two patients following 2 weeks at $60 \mathrm{mg} /$ day, and in two patients following 2 weeks at $90 \mathrm{mg} /$ day.

Of the four patients who received tranylcypromine, three attained a dosage of $40 \mathrm{mg} /$ day for two weeks; one patient was withdrawn from this medication after two weeks on $20 \mathrm{mg} / \mathrm{day}$ when she developed manic symptomatology, further described below. Average dosage for the group receiving tranylcypromine was therefore $35 \mathrm{mg} /$ day; mean duration of treatment was 7 weeks.

Of the two patients who did not complete the study, one reported a dramatic and persistent decrease in symptoms three weeks after discontinuing an unsuccessful 13-week trial of fluoxetine - "probably," said the patient, "because I decided I had had enough of all this worrying and checking and just decided to resist all of the impulses - and, they started going away." His Y-BOCS item 1-10 score fell from an average of 18 during the medication trials to 7.5 after this improvement (week 16). This patient therefore did not go on to trials of either MAOI. The second patient who failed to complete the study dropped out after having intolerable sedation and headache on $30 \mathrm{mg} /$ day phenelzine and an unsuccessful eight-week course on up to $60 \mathrm{mg} /$ day fluoxetine.

Of particular note, one patient developed mild to moderate manic symptomatology (no prior history of mania) on all three medications. Symptoms included decreased need for sleep to about two hours nightly, 
pressured speech, flight of ideas, distractibility, hyperactivity, irritability, and elated mood with frequent euphoria; there were no psychotic features. These symptoms developed after four weeks on $60 \mathrm{mg} /$ day phenelzine; after two weeks on $20 \mathrm{mg} /$ day tranylcypromine; and after two weeks on $20 \mathrm{mg} /$ day fluoxetine, increasing in severity until withdrawn from the medication after two weeks on $60 \mathrm{mg} /$ day. Interestingly, at no time during these episodes did obsessive or compulsive symptoms abate: reported the patient, "Now I just clean faster and find more things to clean, stay up all night fixing and rearranging the house, and obsess more about all of the things I should do . . . but for some reason these things just don't bother me very much anymore!"

Since termination of this study, one of the patients entered formal behavior therapy involving exposure and response prevention (Marks, 1987). After five biweekly sessions, this patient's item 6-10 Y-BOCS decreased to 5.5 from an average of 10.5 during drug treatment (this patient suffered from compulsions only; item 1-5 score was zero). Following unsuccessful trials of all three medications, a second patient began psychotherapy in which she became aware of the sexual nature of her obsessions and was gradually desensitized to the associated feelings of shame and guilt; she also read the book Living with Fear (Marks, 1978) in attempt to learn behavioral techniques for symptom control. This patient reported that both of these treatment modalities helped to reduce her OC symptoms; her Y-BOCS item 1-10 score from an average of 20 during drug-treatment to 10 after three months of psychotherapy and of following the guidelines presented in the book. The patient whose symptoms appeared to improve spontaneously after he resolved simply to resist all obsessional or compulsive impulses was able to maintain this improvement at eight-week follow-up by continuing with this tactic whenever impulses arose anew. Each of these therapeutic approaches share with behaviorally oriented therapy the fundamental feature of gradual desensitization to the distressing affect associated with confrontation of obsessional thoughts or compulsive impulses (Marks, 1987).

\section{DISCUSSION}

Based on the literature review, we began this open-label study with the beliefs that fluoxetine would ameliorate obsessive and/or compulsive (OC) symptoms in most patients and that the MAOIs might be at least partially helpful in reducing OC symptoms. Despite our initial biases, however, we found that none of these agents showed any apparent efficacy in treating the OC symptoms in these patients. The frequent development of medication side effects, and pill-counts that were always in close agreement with dosage instructions, argue against noncompliance with medication regimen as a likely explanation. Given the major clinical improvement in these three patients following this study, it also seems unlikely that our sample consisted of an unusually treatment-refractory 
group of patients. It is possible that some improvement in illness-related emotional or functional disability may have gone undetected by reliance on only one objective measure of treatment outcome (Y-BOCS); however, it seems unlikely that the $\mathrm{Y}-\mathrm{BOCS}$ lacked sufficient sensitivity to detect actual changes in $\mathrm{OC}$ symptoms or related functional impairment since it did detect the subjectively observed changes over time (probable regression toward the mean) and clinical improvement in the three patients whose symptoms decreased following completion of the study.

The small sample-size and methodologic limitations of this study do limit the generalizability of these findings and the ability to answer our initial questions conclusively. We believe, however, that some important inferences may be drawn from our observations.

First, the lack of clinical improvement in any patient from the drugs studied (with treatment sometimes limited by development of intolerable medication side effects) suggests that there may be many patients with OCD for whom these agents - including the serotonergic agent fluoxetine-are ineffective or poorly tolerated. It is certainly possible that effective treatment with these agents may require dosages or treatment-duration exceeding those used in this study. Of note, since the completion of this study, we have had one patient in our clinic who presented with severe obsessive ruminations (OCD) which did not clear until fluoxetine dosage was raised to $120 \mathrm{mg} /$ day; symptoms subsequently recurred within a week following a decrease in dosage to $100 \mathrm{mg} /$ day, then again disappeared with resumption of $120 \mathrm{mg} /$ day fluoxetine. Second, the findings support the effectiveness of behavioral-therapeutic techniques even in patients for whom medications prove ineffective or intolerable (Marks, 1987).

Finally, one patient clearly displayed the coexistence of mild manic episodes and OC symptoms on all three medications. There have been several reports published describing the disappearance of OC symptoms when patients experienced manic episodes or periods of elevated mood (Insel, Murphy, \& Cohen, 1983; Gordon \& Rasmussen, 1987; Kendell \& Discipio, 1970; Stengel, 1945; Turner, Jacob, Beidel, \& Griffin, 1985; White et al., 1986); these reports have prompted the suggestion that the coexistence of these two syndromes may not be possible (Gordon \& Rasmussen, 1987; Kendell \& Discipio, 1970; Stengel, 1945), perhaps because of critical differences in central serotonergic activity between them (Gordon \& Rasmussen, 1987). Our patient did become less botheredand even a bit amused-by her OC symptoms during the episodes of manic symptomatology, but the intensity and frequency of obsessions and compulsions did not diminish. This observation argues against the proposal that these syndromes cannot coexist or are neurochemically incompatible.

\section{REFERENCES}

Folks, D. G. (1983). Monoamine oxidase inhibitors: Reappraisal of dietary considerations. Journal of Clinical Psychopharmacology, 3, 249-252. 
Fontaine, R., \& Chouinard, G. (1986). An open clinical trial of fluoxetune in the treatment of obsessive-compulsive disorder. Journal of Clinical Psychopharmacology, 6, 98-101.

Goodman. W. K., Price. L. H., Rasmussen, S. K., Mazure, C., Delgado, P., Heninger, G. R., \& Charney, D. S. (in press). The Yale-Brown Obsessive-Compulsive Scale (YBOCS): Part II. Validity. Archives of General Psychiatry.

Goodman, W. K., Price, L. H., Rasmussen, S. K., Mazure, C., Fleischmann, R., Hill, C., Heninger, G. R., \& Charney, D. S. (in press). The Yale-Brown Obsessive Compulsive Scale (Y-BOCS): Part 1. Development, use, and reliability. Archives of General Psychiatry.

Gordon, A., \& Rasmussen, S. A. (1987). Mood-related obsessive-compulsive symptoms in a patient with bipolar affective disorder. Journal of Clinical Psychiatry, 49, 27-28.

Insel, T. R., Murphy, D. L., \& Cohen, R. M. (1983). Obsessive-compulsive disorder. A double-blind trial of clomipramine and clorgyline. Archives of General Psychiatry 40, 605-612.

Jenike, M. A. (1981). Rapid response of severe obsessive-compulsive disorder to tranylcypromine. American Journal of Psychiatr. 138, 1249-1250.

Jenike, M. A., Surman, O. S., Cassem, N. H., Zusky, P., \& Anderson. W. H. (1983). Monoamine oxidase inhibitors in obsessıve-compulsive disorder. Journal of Clinical Psychiatry, 44, 131-132.

Kendell, R. E., \& Discipio, W. J. (1970). Obsessional symptoms and obsessional personality traits in patients with depressive illnesses. Psychological Medicine, 1, 65-72.

Mahgoub. O. M. (1987). A remarkable response of chronic severe obsessive-compulsive neurosis to phenelzine. Acta Psychiatrica Scandinavica, 75, 222-223.

Marks, I. M. (1987). Fears, phobias, and rituals. (pp. 228-278. 457-494). New York: Oxford University Press.

Marks, I. M. (1978). Living with fear. New York: McGraw-Hill.

Sommi, R. W., Crismon, M. L., \& Bowden, C. L. (1987). Fluoxetine: A serotonın-specific. second-generation antidepressant. Pharmacotherapy, $7,1-15$.

Stengel, E. (1945). A study on some clinical aspects of the relationship between obsessional neurosis and psychotic reaction types. Britush Journal of Psychiatry, 91, 166-187.

Turner, S. M., Jacob, R. G., Beidel, D. C., \& Griffin, S. (1985). A second case of mania associated with fluoxetine. American Journal of Psychiarry, 142, 274-275.

Turner, S. M., Jacob, R. G., Beidel, D. C., \& Himmelhoch, J. (1985). Fluoxetine treatment of obsessive-compulsive disorder. Journal of Clinical Psychopharmacology, 5, 207-212.

White, K., Keck, P. E., \& Lipinski, J. (1986). Serotonin-uptake inhibitors in obsessivecompulsive disorder: A case report. Comprehensive Psychiatry, 27, 211-214. 